\title{
A Survey on Mobility based Routing Protocols in Wireless Sensor Networks
}

\author{
Krishna K.K. \\ Toc $\mathrm{H}$ Institute of Science and Technology, \\ Arakkunnam \\ Ernakulam, Kerala-682315
}

\begin{abstract}
A wireless sensor network is consisting of multifunctional sensor nodes scattered in sensing fields. There are many routing protocols in the field wireless sensor networks. Routing protocols are use to extend the life of the wireless sensor networks with efficient data delivery. The mobility that greatly affects the performance of routing protocols in the wireless sensor networks. This paper presents review of the five mobility based routing protocols for WSNs. The mobility based protocol can improve the network lifetime of wireless sensor networks.
\end{abstract}

\section{Keywords}

Wireless sensor networks, Routing protocols, Sink mobility

\section{INTRODUCTION}

Wireless sensor network is a wireless network made up of spatially distributed autonomous devices to monitor physical or environmental conditions at various locations. The various application of WSN is in military area, environment area, health, home and other commercial areas [1].Routing in WSN is different from conventional routing .Many routing protocols were developed for wireless networks[2]. The mobility that greatly affects the performance of routing protocols in the wireless sensor networks. The sensor nodes find the route towards to transmit the data. The sensor node transmits the data directly or relays the data through neighbor nodes to mobile sinks. The nodes close to the sink node transmit more data than other nodes. It results in the small lifetime of the network. To increase the lifetime of the network, use the mobile sink node approach. The mobile sink creates the network dynamic. It is a challenging job to find the route in the dynamic network.

In this paper, focus on mobility based routing protocols in wireless sensor networks. Each routing protocol has its own advantages and disadvantages. The rest of the paper is structured as follows. In Section 2 present the network design challenges and routing issues. In Section 3 present most popular mobility based routing protocols in wireless sensor networks. Finally, Section 4 draws the main conclusion of this work.

\section{NETWORK DESIGN CHALLENGESAND ROUTING ISSUES}

The major network design challenges and routing issues [3] are listed below:

- $\quad$ Limited energy capacity: A sensor node has limited battery capacity. The routing protocols designed for sensors should be energy efficient. The energy efficient sensor nodes improve the network lifetime.

\author{
Rani Augustine \\ Toc $\mathrm{H}$ Institute of Science and Technology, \\ Arakkunnam \\ Ernakulam, Kerala-682315
}

- Sensor locations: The one of the design challenges of routing protocols is to manage the locations of the sensors. Sensor node deployed in any area can monitor and trace its location by the base station.

- Limited resources: The network resources such as memory, power supply, and bandwidth are limited.

- Diverse sensing application requirements: The network protocol cannot meet all the requirements of all applications. So develop the routing protocols should guarantee efficient data delivery and high accuracy.

- Scalability: Routing protocols should be able to scale with the network size.

- Mobility Issues: Most of the sensor nodes are mobile in nature. But some problems occur while sink node is also mobile in nature. The energy-sink holes problems occur by using static sink node.

\section{MOBILITY BASED ROUTING \\ PROTOCOLS}

Mobility creates new challenges to routing protocols in WSNs. Sink mobility requires energy efficient protocols for secure data delivery originated from source sensors toward mobile sinks. Some of the protocols in case mobility based routing are as below:

- Scalable Energy -Efficient Asynchronous Dissemination( SEAD)

- $\quad$ Two-Tier Data Dissemination (TTDD)

- Data MULES

- Dynamic Proxy Tree-Base Data Dissemination

- Tree-Based Efficient Data Dissemination Protocol( TEDD)

\subsection{Data Mules}

Basic operation:

This protocol was proposed to concentrate on the need of reliable and cost effective connectivity in sparse network by reducing energy consumption[4]. It is a three-tier architecture based on mobile entities, called mobile ubiquitous LAN extensions (MULE).The architecture of MULE consists of three layers. The lower layer contains static wireless sensor nodes whose duty is to sense the environment. The top layer includes WAN connected units and access points. These access points communicates with central data warehouse and enabling them for synchronization of collected data, identification of redundant data and acknowledgment of data send by MULEs for reliable data transmission. Middle layer has mobile units(MULEs) that move across 
sensor field and gather data from sensor nodes and deliver to access point which are in close range.

\section{Advantages}

- Achieve cost-effective connectivity in sparse sensor networks.

- Data transfers only occur at short distances leading to power savings.

- Fewer infrastructures are needed.

Disadvantages

- Latency for this type of network is high.

- Encounter unexpected failures such as loss of a MULE or inability to reach sensors because of change in terrain causing limitations in mobility.

\subsection{Scalable Energy-Efficient Asynchronous Dissemination}

Basic operation:

SEAD was Self-organizing protocol which focus on minimization of delay to sink node and savings of energy level[5]. SEAD uses data dissemination concept in which sensor nodes reports its sensed data to multiple sink node which come under its range. It consists of three main steps namely dissemination tree construction (d-tree), data dissemination and maintenance of links to mobile sink nodes. It consider a assumption that all sensor nodes have an idea about their geographic location. All sensor node build its d-tree rooted at itself and for all nodes it is built separately. This protocol is also categorized as location aware routing protocols.

Advantages

- It distributes most of the data successfully.

- It minimizes the energy cost

Disadvantage

- $\quad$ Packet delivery delay to the sink increases.

\subsection{Dynamic Proxy Tree-Based Data Dissemination}

Basic operation

A dynamic proxy tree-based data dissemination protocol [6] was proposed for maintaining a tree connecting a source sensor to multiple sink nodes that are interested in the source. This will helps the source node disseminate its data directly to those mobile sink nodes. In this protocol, a network is consists of stationary sensors and several mobile sinks. The sensors are used to detect and monitor some mobile targets, where the mobile sinks are used to collect data from specific sensors which may detect the target and periodically generate detected data or aggregate detected data from a subset of sensors. Due to target mobility, a source node may change and a new sensor closer to the target node may become a source node. Each source node is represented by a static source proxy and each sink is represented by a static sink proxy. The source and sink proxies are change as the source sensors change and the sink nodes move. A new source proxy creates for a source only when the distance between the source and its current proxy exceeds a certain threshold. Then a sink will have a new sink proxy only when the distance between the sink and its current proxy exceeds a certain threshold.
Advantages

- Design of proxies reduces the cost of transmitting data from the source and sinks proxies.

Disadvantages

- Large flooding overhead.

- High packet delivery delay.

\subsection{Two-Tier Data Dissemination Protocol} Basic operation:

TTDD, a Two-Tier Data Dissemination protocol [7] that provides scalable and efficient data delivery to multiple, mobile sinks. Each data source node in TTDD proactively constructs a grid structure, which helps mobile sinks to continuously get data on the move by flooding queries within a local cell only. Two-Tier Data Dissemination protocol design exploits the fact that sensors are static and location-aware to build and maintain the grid infrastructure with low overhead. The data source node proactively builds a grid structure throughout the sensor field and sets up the forwarding information at the sensors nearest to grid points. This grid structure in place, a query from a sink traverses two tiers to reach a source. The bottom tier is within the local grid square of the sink node current location and the top tier is made of the dissemination nodes at grid points. The sink node floods its query within a cell. When the closest dissemination node for the requested data receives the query then node forwards the query to its upstream dissemination node toward the source node, which in turns further forwards the query, until it reaches either the source or a dissemination node that is already receiving data from the source node. This query forwarding process places information of the path to the sink node, to helps data from the source to traverse the same two tiers as the query but in the reverse order.

Advantage

- Increase energy efficiency and decrease the latency due to their connectivity capability.

- $\quad$ Fewer infrastructures are needed.

Disadvantage

- Packet delivery delay occurs.

\subsection{Tree-Based Efficient Data Dissemination Protocol \\ Basic Operation:}

Tree- based efficient data dissemination protocol [8] with mobile sink. First, it creates the tree in the network with a root node. There are two types of the nodes in the network. They are relay node and non-relay node. The relay node is responsible for relay the data from the nodes to its next relay node. The non-relay node can only transmit its data to a relay node. It is a unidirectional communication between non-relay and relay nodes. The communication is bidirectional between two relay nodes. The tree topology varies when the role of the node varies from relay to non-relay or from non-relay to relay node. The sink node is mobile and gathers the data from the source nodes through the gateway node. The gateway node may be the relay node or the non-relay node. The sink periodically passes a small beacon to make the connection alive with the gateway node. The sink moves out from the range of the current gateway node, and then it selects another node as the gateway node. 


\section{Advantages}

- Throughput is very high.

- Less control packet overhead.

Disadvantage

- $\quad$ Needs more memory for tree construction and sink management.

\section{CONCLUSION}

Routing in wireless sensor networks is a new area of research, with a limited, but rapidly growing set of research results. In this paper, presented comprehensive survey mobility based routing protocols in wireless sensor networks along with their advantages and disadvantages. The main common objective is to prolong the lifetime of the sensor network, without compromising data delivery by using knowledge about mobility of sink nodes. However, many of these mobility based routing protocols look promising, but still many challenges that need to be solved in the sensor networks. The future scope of this study is to develop efficient routing protocols based on mobility of sink nodes.

\section{REFERENCES}

[1] F. Akyildiz, W. Su, Y, Sankarasubramaniam, and E. Cayirci, "Wireless sensor networks: A survey," Comput. Netw., vol. 38, no. 4, pp. 393-422, Mar. 2002
[2] Jun Zheng and Abbas Jamalipour, "Wireless Sensor Networks: A Networking Perspective", a book published by A John \& Sons, Inc, and IEEEE, 2009.

[3] Gowrishankar.S, T.G.Basavaraju , ManjaiahD.H 3 , Subir Kumar Sarkar ," Issues in Wireless Sensor Networks", Proceedings of the World Congress on Engineering 2008 Vol I WCE 2008, July 2 - 4, 2008 , London, U.K

[4] R.C. Shah, S. Roy, S. Jain, and W. Brunette, "Data MULEs: Modeling a three-tier architecture for sparse sensor networks ", Proceedings SN P A '03, Anchorage, AK, May 2003, pp. 30-41.

[5] H. S. Kim, T. F. Abdelzaher, and W. H. Kwon,"Minimum-energy asynchronous dissemination to mobile sinks in wireless sensor networks",In ACM SenSys, 2003.

[6] W. Chang, G. Cao, and T. La Porta, "Dynamic proxy tree-based data dissemination schemes for wireless sensor networks", Proceedings IEEE MASS'04, Fort Lauderdale, FL, Oct. 2004, pp. 21-30.

[7] Haiyun Luo,Fan Ye, Jerry Cheng, Songwu Lu, Lixia Zha ng," TTDD: Two-Tier Data Dissemination in Large-Scale Wireless Sensor Networks"

[8] Suraj, Sharma,Sanjay Kumar Jena"Data Dissemination Protocol for Mobile Sink in Sensor Networks", Journal of Computational Engineering Volume 2014 (2014), Article ID 560675, 10 pages 